\title{
The history of the International Astronomy Olympiad
}

\author{
Michael G. Gavrilov ${ }^{1}$, Igor V. Salnikov ${ }^{2}$ and Anders R. Vaesterberg ${ }^{3}$ \\ ${ }^{1}$ ISSP of Russian Academy of Sciences, \\ Institute street 2, 142432 Chernogolovka, Moscow region, Russia \\ email: gavrilov@issp.ac.ru \\ ${ }^{2} 2$ Simferopol Society of Amateur Astronomers, \\ Simferopol, Crimea, Ukraine \\ email: solacom@ukr.net \\ ${ }^{3} 3$ European Association for Astronomy Education, \\ Swedish Branch, Stockholm, Sweden \\ email: vaesterberg@hotmail.com
}

\begin{abstract}
The International Astronomy Olympiad (IAO) was founded in the 1990s as an annual scientific educating event for students of the junior high school classes. Starting from 4 teams at the 1st event in 1996 the Olympiad includes more than 20 countries nowadays. The style of the problems of IAO is aimed at developing the imagination, creativity and independent thinking. They stimulate the students to recognize the problem independently, to choose a model, to make necessary suppositions, estimations, to conduct multiway calculations or logic operations. The Asian-Pacific Astronomy Olympiad was founded as a "daughter" ("affiliated") olympiad in system of the International Astronomy Olympiad in 2005.
\end{abstract}

Keywords. Olympiad, education, teaching

\section{The International Astronomy Olympiad}

The International Astronomy Olympiad is a scientific-educating event for students of the junior high school classes, aged 14-18 years, and which includes an intellectual competition between these students. The style of the problems is aimed at developing the imagination, creativity and independent thinking $\dagger$ The Olympiad is carried out in the spirit of friendship, tolerance, where the competition is a stimulus for showing the participants' capabilities but contacts, exchange of ideas and collaboration between students, teachers and scientists from various countries have a primary importance. It should be emphasized that the competition at the International Astronomy Olympiad is not the most important part of the programme, in other words the competition with its points, places and Diploma is only a tool but not the purpose of the Olympiad, and the Olympiad is not a "selection of the best students" nor an "examinations".

The IAO takes place each year in the first two months of the astronomical autumn (i.e. September 22 - November 22) in either an observatory, a scientific research centre (town) or at an institute of the participating countries. The IAO is organised by the Authorized National Representative (Astronomical) Organization (ANRAO) of the organizing country.

The Olympiad is intended for students of the adolescent age when the interest to astronomy is being formed most actively. Then preparation for participation is still a

$\dagger$ see an example in M.G. Gavrilov's contribution in this volume (page 720). 
useful and purposeful education not yet having turned training into an end in itself. This is the main idea of the Olympiad. A considerable part of the knowledge which is necessary for participation in the Olympiad goes beyond the framework of the school curriculum in most of the countries in the world. Preparation for the Olympiad needs therefore extracurricular activities of various types, whose promotion is one of the Olympiad's aims.

The total amount of knowledge is comparatively not too large and acquiring it is completely within the reach of the students of the above-mentioned age. It is also the age for which the spirit of the Olympiad as an entertaining competition is most appropriate.

Involving students of the senior classes would lead to enlarging and complicating the necessary material, which would be senseless. For such students it already turns into a kind of sport and is significantly less useful from an educational point of view. For students aged 17-20 more serious events are preferable like conferences and competitions of research projects.

The International Olympiad is also a meeting of teachers and scientists, where exchange of ideas takes place, methods for refining the astronomical education are discussed and international collaboration in this field is established.

\section{The beginnings of the IAO}

Competitions for young amateur astronomers, called "Olympiads", were carried out in former USSR fo many years. The first attempts to organise a competition at the international (i.e. multilanguage) scale started in the scientific town of Soviet Academy of Sciences "Chernogolovka" in 1990-1991 as part of the so called Olympiad of Naukograds and Scientific Centres (NSC Olympiad). Teams of Russia, Moscow, Latvia and Estonia participated. These events provided ground for the future IAO proper.

Positive experience during international olympiads in other natural sciences (Physics, Chemistry, Biology), Mathematics and Informatics led to the idea of starting an International Astronomy Olympiad.

Juridical founding of the International Astronomy Olympiad has been done by EuroAsian Astronomical Society on June, 7, 1996, as an annual astronomy competition for high-school students. Immediately after the founding the Olympic Coordinating Council was established. The first official IAO was held in the Special Astrophysical Observatory of Russian Academy of Sciences in November 1996 as a part of programme of the Autumn Astronomy School for Young Astrophysists. Notwithstanding some initial difficulties, this olympiad was a great success and it was decided to continue with the IAO. In subsequent olympiads the number of participating states increased. So the Olympiad of Naukograds and Scientific Centres and Autumn Astronomy School of Young Astrophysists in the Special Astrophysical Observatory may be considered as father and mother of the International Astronomy Olympiad.

\section{Aims of the IAO}

In recognition that:

- astronomy plays a fundamental rôle in the human progress and will acquire more global importance in the 21 century; 
- astronomical knowledge is an important part of the culture of our civilisation and an essential factor in forming the view of life and the way of thinking of the young people in the modern world;

The Olympiad sets itself the aims of:

(a) Attracting the most talented young people to professional careers in the field of astronomy:

- giving an opportunity for showing their capabilities and encouraging the best students;

- helping the young people in choosing a profession;

- acquainting the participants with the real working conditions and the nature of the research work in the hosting observatory (institute), exchange of ideas and knowledge between astronomers, teachers and students.

(b) Spreading astronomical knowledge among as many students as possible and improving the astronomical education:

- provoking an interest in astronomy, physics and astronautics in a greater number of children and young people;

- popularisation of natural science and the scientific approach in astronomy and related sciences;

- encouraging teachers to work for improving, enriching and enlarging school astronomy education and including more children in it;

- activating the astronomy education in the junior classes of the high school; promoting extracurricular activities in amateur clubs, scientific societies, circles etc.;

- stimulating organization of national astronomical Olympiads in different countries;

- enhancing international contacts in the field of astronomy and physics education in schools.

(c) Stimulating the imagination and creativity of children:

- the character of the astronomical tasks enables putting the students in non-standard situations, very close to those of a real scientific research; they can require creating hypotheses, assuming approximations, choosing between a multitude of factors that could be taken into account or neglected, and independent decision about the form of the needed answers;

- the astronomical tasks can set unusual problems for which solving a free way of thinking, fantasy and inventiveness are necessary;

- astronomical tasks allow a variety of completely different, although correct solutions applying different original approaches.

(d) Cultivating a spirit of correctness and friendship:

- the International Astronomy Olympiad is a meeting of young people from different countries -future colleagues in the scientific exploration who will have to work in cooperation;

- in the time of the Olympiad favourable conditions are created for active contacts between students, teachers and specialists from the hosting observatory (institute) aimed at an intense exchange of knowledge, education and upbringing of the children during all stages of the event. 